PROCEEDINGS OF THE

AMERICAN MATHEMATICAL SOCIETY

Volume 137, Number 5, May 2009, Pages 1813-1821

S 0002-9939(08)09695-0

Article electronically published on December 15, 2008

\title{
ON THE LINEARITY OF TORSION-FREE NILPOTENT GROUPS OF FINITE MORLEY RANK
}

TUNA ALTINEL AND JOHN S. WILSON

(Communicated by Julia Knight)

\begin{abstract}
It is proved that every torsion-free nilpotent group of finite Morley rank is isomorphic to a matrix group over a field of characteristic zero.
\end{abstract}

\section{INTRODUCTION}

The central problem in the analysis of groups of finite Morley rank has been the classification of the infinite simple groups of finite Morley rank; these were conjectured independently by Gregory Cherlin and Boris Zil'ber to be linear algebraic groups over algebraically closed fields. The determination of sufficient conditions for groups of finite Morley rank to be linear seems an important question in its own right. In view of the linear behaviour of soluble groups, it seems natural to expect that many groups of finite Morley rank are linear; indeed we know of no counter-examples to the following:

Conjecture. If $G$ is a connected soluble group of finite Morley rank, then $G$ has a central subgroup $Y$ such that $G / Y$ can be embedded in a direct product of finitely many linear groups over fields.

Substantial progress has been made recently by Frécon [6], who proved that if $G$ is a connected soluble group of finite Morley rank with trivial centre and the second derived group of $G$ is torsion-free, then $G$ behaves in accordance with the conjecture; indeed, $G$ is definably isomorphic to a subgroup of $\mathrm{GL}_{n}\left(F_{1} \oplus \cdots \oplus F_{m}\right)$ for some integer $n$ and some algebraically closed fields $F_{1}, \ldots, F_{m}$.

Here we give further evidence for the conjecture:

Theorem 1. Every torsion-free nilpotent group $G$ of finite Morley rank has a faithful linear representation over a field of characteristic 0 .

We prove Theorem 11 by using an adaptation of a linearization technique for algebras introduced by Zil'ber in [14. This is applied to the Lie algebras in characteristic 0 obtained from our groups using the Maltsev correspondence (see Section 2 below).

Received by the editors March 3, 2008, and, in revised form, July 9, 2008.

2000 Mathematics Subject Classification. Primary 03C60, $20 \mathrm{~F} 16$.

The first author was supported by MODNET, an FP6 Marie Curie Research Training Network in Model Theory and its Applications, funded by the European Commission under contract number MRTN-CT-2004-512234 (MODNET).

(C)2008 American Mathematical Society Reverts to public domain 28 years from publication 
We are unable to make a definability assertion like the one in Frécon's result. This is not altogether surprising in our context of nilpotent groups. At certain points splitting problems arise, and in avoiding them we lose definability. An intriguing question, raised by Frécon, is whether there exist not necessarily definable representations of our groups $G$, whose images are nevertheless definable subgroups of general linear groups.

We note that an analysis of nilpotent groups of finite Morley rank that are not torsion-free seems likely to be hard, as witnessed by the construction by Baudisch [2] of nilpotent groups of finite Morley rank of bounded exponent that do not interpret fields.

\section{Preliminaries}

In this section we record some preliminary results. Our references for groups of finite Morley rank and for general model theory are [3] and 7].

Groups of finite Morley rank do not have infinite strictly descending chains of definable subgroups, a fact that we will refer to as the descending chain condition or the minimal condition on definable subgroups. As a consequence of this finiteness condition, each group $G$ of finite Morley rank has a unique smallest definable subgroup of finite index, called the connected component of $G$ and denoted by $G^{\circ}$; if $G=G^{\circ}$, then $G$ is said to be connected. It can be shown using relatively elementary methods that torsion-free groups of finite Morley rank are divisible. Thus, they have no proper subgroups of finite index, and they are connected.

The following fact includes some of the corollaries of what is known as Zil'ber's indecomposability theorem. We will refer to the first corollary simply as Zil'ber indecomposability. The second one implies immediately that the derived group of a group of finite Morley rank is definable.

Fact 2.1 ([3, Section 5.4]). Let $G$ be a group of finite Morley rank. Then

(a) the subgroup generated by a set of definable, connected subgroups of $G$ is definable and it is the setwise product of finitely many of these definable connected subgroups; and

(b) if $H$ is a definable, connected subgroup of $G$ and $X$ is an arbitrary subset, then the subgroup $[H, X]$ is definable and connected.

We shall prove a short technical lemma that combines a result of Cohen [5] with various results on the model theory of rings. Before stating it, we recall that the notion of connectedness can naturally be defined in rings of finite Morley rank. Indeed, if $R$ is a ring of finite Morley rank, then its connected component $R^{\circ}$ is defined as the connected component of the additive group $(R,+)$. It is immediate (see [3, p. 79]) that $R^{\circ}$ is in fact a two-sided ideal.

Lemma 2.2 (cf. 4], 11], 14]). Let $\Lambda$ be a connected commutative local ring of finite Morley rank, with maximal ideal $J$. Then $\Lambda / J$ is an algebraically closed field. If in addition $\Lambda / J$ has characteristic 0 , then there is a field $\mathfrak{K} \leq \Lambda$ such that $\Lambda=J+\mathfrak{K}$ and $J \cap \mathfrak{K}=\{0\}$. Moreover $\Lambda$ is finite-dimensional, regarded as a vector space over $\mathfrak{K}$.

Proof. Since $\Lambda$ is a local ring, $J$ is exactly the Jacobson radical of $\Lambda$. It follows from the basic structure theory of rings (see for example [9, Proposition 4.5 (2), 
p. 195]) that the Jacobson radical is definable in the ring language by the formula

$$
\forall y \exists z(y x+z-z y x=0) .
$$

It is also known (see any of the references in the statement or [3, Appendix A]) that the Jacobson radical in a ring of finite Morley rank is nilpotent. Thus, for some $m$ we have $J=\left\{\lambda \in \Lambda \mid \lambda^{m}=0\right\}$. Being an infinite field of finite Morley rank, $\Lambda / J$ is algebraically closed (see [10]). Therefore from Cohen [5, Theorem 9], there is a field $\mathfrak{K} \leq \Lambda$ such that $\Lambda=J+\mathfrak{K}$ and $J \cap \mathfrak{K}=\{0\}$. By Zil'ber indecomposability, each of the powers $J^{i}$ is a finitely generated $\Lambda$-module. Hence each $J^{i} / J^{i+1}$ is finitely generated as a module for $\Lambda / J$ or equivalently for $\mathfrak{K}$, and so $\Lambda$ has finite dimension over $\mathfrak{K}$.

The Maltsev correspondence associates with each divisible torsion-free nilpotent group $G$ a nilpotent Lie algebra $L$ over the field $\mathbb{Q}$. Our references for the Maltsev correspondence are Stewart [12] and Warfield [13]. In [12, the correspondence is set up in such a way that $L$ coincides with $G$ as a set, and the identity map from $G$ to $L$ induces an order-preserving bijection from the set of divisible subgroups to the set of Lie subalgebras in which normal subgroups correspond to ideals.

We recall that if an arbitrary nilpotent group has an element of order $n>1$, then it has a non-trivial central element of order dividing $n$; we also recall that torsion elements are central in divisible nilpotent groups ([13, Chapters 2-3]). It follows from these facts that elements of a divisible torsion-free nilpotent group $G$ have unique $s$ th roots for all integers $s \neq 0$; thus for $g \in G$ and $\lambda \in \mathbb{Q}$, the power $g^{\lambda}$ can be uniquely defined by the equation $\left(g^{\lambda}\right)^{s}=g^{r}$, where $\lambda=r / s$ with $r, s \in \mathbb{Z}$ and $s \neq 0$.

Lemma 2.3 (see [12]). Let $G$ be a divisible torsion-free nilpotent group of class $c$. Let $L$ be the Lie algebra associated with $G$ via the Maltsev correspondence. Then the following assertions hold:

(a) $Z(G)=\operatorname{Ann}(L)$ and $G^{\prime}=L^{2}$;

(b) definable central subgroups of $G$ correspond to definable subalgebras of $\operatorname{Ann}(L)$;

(c) G has finite Morley rank if and only if L has finite Morley rank.

Proof. In [12, Section 2], the Hausdorff-Campbell formula is used to show how the Lie algebra operations for $L$ can be defined from the group operation on $G$. The results in [12] show that for certain words $\sigma_{c}(x, y), \pi_{c}(x, y)$ in the free group on $x, y$, the sum and Lie product in $L$ can be defined by $u+v:=\sigma_{c}(u, v)$ and $[u, v]:=$ $\pi_{c}(u, v)$ for all $u, v \in G$; multiplication by rationals $\lambda$ is defined by $\lambda u:=u^{\lambda}$. Here, $c$ is the nilpotency class of $G$; because we are working with a nilpotent group, it is not necessary to consider the 'extended words' $\sigma, \pi$ constructed in [12].

We also need to consider how $G$ may be defined in terms of the Lie algebra $L$. It is shown in 12 that there is a word $\mu(x, y)$ in the free Lie algebra on $x, y$ such that products and inverses are defined in $G$ by $u v:=\mu(u, v)$ and $u^{-1}:=-u$.

The first part of Assertion (a) is [12, Corollary 2 to Theorem 2.4.2]. The second part follows from the definitions of [,] and (, ) in [12, Lemma 2.3.1]. Indeed, from the definition of [,] one deduces that $L^{2} \subseteq G^{\prime}$, and from the definition of (,) it follows that $G^{\prime} \subseteq L^{2}$. It is also clear that subgroups of $G$ correspond to subalgebras of $L$.

If $X$ is a subset of $L$ defined by a formula $\phi$ in the language of Lie algebras, then in $\phi$ we can replace each of the Lie algebra operations by group operations using 
$\sigma, \pi$; it follows that $X$ is also a definable subset of $G$. A similar argument now shows that the subsets definable in $G$ are precisely the subsets definable in $L$, and (b) follows.

The arguments above show that the structure $L$ is definable in the structure $N$, and conversely. Thus $L$ has finite Morley rank if and only if $G$ has finite Morley rank (see for example [7, Theorem 5.6.11]).

Now suppose that $\mathfrak{K}$ is an arbitrary field of characteristic 0 . If $L$ is a Lie algebra over $\mathfrak{K}$ and $G$ is the group associated with $L$ by the Maltsev correspondence as above, then for all $u$ in $N$ and $\lambda$ in $\mathfrak{K}$ we may define the power $g^{\lambda}$ in $G$ to be the multiple $\lambda g$ in $L$. The map $G \times \mathfrak{K} \rightarrow G$ defined in this way satisfies the axioms characterizing $\mathfrak{K}$-groups (or $\mathfrak{K}$-powered groups), described for example in Warfield [13. Chapters 10-12]. Up to isomorphism, all $\mathfrak{K}$-groups arise in this way, and so the Maltsev correspondence specializes to give a correspondence between $\mathfrak{K}$-groups and Lie algebras over $\mathfrak{K}$.

\section{ZIL'BER'S LINEARIZATION CONSTRUCTION}

The following general construction is described in [14, Theorem 3].

Let $R$ be a (not necessarily associative) $\mathbb{Q}$-algebra of finite Morley rank such that $R^{2} \neq 0$. Write $A=\operatorname{Ann}(R)$ and $\bar{R}=R / A$. We shall show that a certain ring of group endomorphisms of $\bar{R}$ is interpretable in $\bar{R}$.

Fix a finite set $S$ with $A=\operatorname{Ann}(S)$, say $S=\left\{s_{1}, \ldots, s_{l}\right\}$. Such a set $S$ exists from the descending chain condition for definable subgroups of $(R,+)$.

We consider the set $R^{(l)}$ of $l$-tuples of elements of $R$; we say that two elements of this set are 'congruent mod $A$ ' if their images in $\bar{R}^{(l)}$ are equal. For each $l$-tuple $u=\left(u_{1}, \ldots, u_{l}\right)$ define

$$
M(u, x, y)=\left(\bigwedge_{i=1}^{l}\left(u_{i} x=s_{i} y\right)\right) \wedge\left(\bigwedge_{i=1}^{l}\left(x u_{i}=y s_{i}\right)\right) .
$$

Write

$$
\Phi_{1}(u)=\forall x \exists y(M(u, x, y)) \quad \text { and } \quad \Lambda_{1}=\left\{u \mid \Phi_{1}(u)\right\} .
$$

For any $u, u^{\prime} \in \Lambda_{1}$ and $x, x^{\prime}, y, y^{\prime} \in R$ we have

- $\left(M(u, x, y) \wedge M\left(u, x, y^{\prime}\right)\right) \rightarrow y-y^{\prime} \in A$;

- $\left(M(u, x, y) \wedge x-x^{\prime} \in A\right) \rightarrow M\left(u, x^{\prime}, y\right)$

- $\left(u, u^{\prime}\right.$ congruent $\left.\bmod A \wedge M(u, x, y)\right) \rightarrow M\left(u^{\prime}, x, y\right)$.

Hence, for each congruence class of elements $u$ of $\Lambda_{1}$, we obtain a map from $\bar{R}$ to itself defined by

$$
\bar{u}(\bar{x})=\bar{y} \text { if and only if } M(u, x, y) .
$$

Clearly each $\bar{u}$ is a group homomorphism. The zero and identity maps are induced by the $l$-tuples $\overline{0}=(\overline{0}, \ldots, \overline{0})$ and $\overline{1}=\left(\overline{s_{1}}, \ldots, \overline{s_{l}}\right)$ respectively.

Write

$$
\Phi_{2}(u)=\forall x, y \in R(\bar{u}(\bar{x} \bar{y})=\bar{u}(\bar{x}) \bar{y}=\bar{x}(\bar{u}(\bar{y}))) ; \quad \Lambda_{2}=\left\{u \in \Lambda_{1} \mid \Phi_{2}(u)\right\}
$$

and

$$
\Phi_{3}(u)=(\forall x)\left(\forall \bar{v} \in \Lambda_{2}\right)(\bar{u}(\bar{v}(x))=\bar{v}(\bar{u}(x))) .
$$

By Zil'ber indecomposability, for some $n$ and $r_{1}, \ldots, r_{n} \in R$ we have $R^{2}=$ $r_{1} R+\cdots+r_{n} R$. (The characteristic 0 assumption implies that each $r_{i} R$ is divisible.) 
Write

$$
\begin{aligned}
\Phi_{4}(u)= & \left(\forall x_{1}, \ldots x_{2 n} \forall y_{1}, \ldots y_{2 n} \forall z_{1}, \ldots z_{2 n}\right) \\
& \left(\left(\sum_{i=1}^{2 n} x_{i} y_{i}=0 \wedge \bigwedge_{i=1}^{2 n} \bar{z}_{i}=\bar{u}\left(\bar{x}_{i}\right)\right) \rightarrow \sum_{i=1}^{2 n} z_{i} y_{i}=0\right)
\end{aligned}
$$

and

$$
\Phi_{5}(u)=\forall x, y, t, r(\bar{t}=\bar{u}(\bar{x}) \wedge \bar{r}=\bar{u}(\bar{y}) \rightarrow t y=x r) .
$$

Finally, define

$$
\Lambda=\left\{\bar{u} \mid \Phi_{i}(u), 1 \leq i \leq 5\right\} .
$$

The following assertions then hold; the first two are clear, and the third is proved in [14, p. 101].

(1) $\overline{0}, \overline{1} \in \Lambda$.

(2) If the elements $u=\left(u_{1}, \ldots, u_{l}\right)$ and $v=\left(v_{1}, \ldots, v_{l}\right)$ of $R^{(l)}$ satisfy $\Phi_{1} \wedge \Phi_{2} \wedge$ $\Phi_{3} \wedge \Phi_{4} \wedge \Phi_{5}$, then so does $w=\left(u_{1}-v_{1}, \ldots, u_{l}-v_{l}\right)$, and $\bar{u}(\bar{x})-\bar{v}(\bar{x})=\bar{w}(\bar{x})$ for all $x \in R$. Therefore $\Lambda$ is an additive subgroup of the ring of group endomorphisms of $\bar{R}$.

(3) For $u$ and $v$ in $R^{(l)}$, the product $\bar{u} \bar{v}$ is defined by an element $w \in R^{(l)}$ where $w=\left(w_{1}, \ldots, w_{l}\right)$ and $\bar{v}\left(\bar{u}_{i}\right)=\bar{w}_{i}$. The operator $w$ thus obtained is in $\Lambda$.

Note that $\Phi_{3}$ ensures that $\Lambda$ is commutative and that $\Lambda$ has finite Morley rank since it is interpretable in $R$. Therefore the arguments above give the following result:

Fact 3.1 ([14, Lemma 3.1]). If $R, \Lambda$ are as above, then $\Lambda$ is a commutative ring of finite Morley rank.

It follows from Lemma 2.2 that $\Lambda$ is a finite-dimensional $\mathfrak{K}$-algebra for some algebraically closed field $\mathfrak{K}$ (see Claim $1(\mathrm{~b})$ below). In the categorical context of [14, the rings $R$ of interest satisfy $A \leq R^{2}$, and using this condition Zil'ber was able to prove that $R$ too has the structure of a finite-dimensional $\mathfrak{K}$-algebra. Though this condition need not hold in our context, we can prove the following result; it is easy to see that the conclusion can hold only if the cardinality hypothesis holds.

Proposition 3.2. Let $R$ be a (not necessarily associative) $\mathbb{Q}$-algebra with $R^{2} \neq 0$. Suppose that $R$ is of finite Morley rank, that $A /\left(A \cap R^{2}\right)$ either is zero or has the same cardinality as $R / A$ where $A=\operatorname{Ann}(R)$, and that $R$ has a unique minimal definable ideal. Then there is an algebraically closed field $\mathfrak{K}$ of characteristic 0 such that $R$ is a finite-dimensional $\mathfrak{K}$-algebra.

Proof. Our first claim is closely related to [14, Lemma 3.7].

\section{Claim 1.}

(a) $\Lambda$ is a local ring.

(b) $\Lambda$ has a subfield $\mathfrak{K}$ such that $\Lambda$ is finite-dimensional, regarded as a vector space over $\mathfrak{K}$.

(c) $\bar{R}$ is finitely generated, regarded as a $\Lambda$-algebra.

Proof of Claim 1. Let $\lambda \in \Lambda$ and suppose that $\lambda$ is non-nilpotent. For each positive integer $m$ the set $\lambda^{m} R$ is a definable ideal; and by the minimal condition on definable subgroups we must have $\lambda^{m} R=\lambda^{m+1} R \neq 0$ for some $m$. Therefore $\lambda$ induces a surjective map from $\lambda^{m} R$ to itself, and so this map must be bijective, 
as otherwise the image would have smaller Morley rank, but in characteristic 0 non-trivial finite kernels are not possible. We conclude that the kernel of the map $r \mapsto \lambda r$ is disjoint from $\lambda^{m} R$ and so is trivial from our hypothesis. Hence $\lambda$ is invertible.

Since $\Lambda$ is commutative, we conclude that the set $J$ of nilpotent elements is the unique maximal ideal of $R$, and (a) follows. Assertion (b) now follows directly from Lemma 2.2. and (c) then follows by Zil'ber indecomposability.

Now, following Zil'ber 14, we make the $\mathbb{Q}$-algebra $R^{2}$ into a finitely generated $\Lambda$-module. We have noted that each element $a \in R^{2}$ can be written in the form $\sum_{i=1}^{n} x_{i} y_{i}$. Let $\lambda \in \Lambda$ and write $\bar{z}_{i}=\lambda \bar{x}_{i}$ for each $i$. We define $\lambda a=\sum_{i=1}^{n} z_{i} y_{i}$. The next claim essentially consists of assertions (a), (b) of [14, Remark 3.3] and the comment before this remark.

\section{Claim 2.}

(a) The value of $\lambda a$ is well defined.

(b) If $a=\sum_{i=1}^{n} x_{i} y_{i}$ and $\bar{t}_{i}=\lambda \bar{y}_{i}$ for each $i$, then $\lambda a=\sum_{i=1}^{n} x_{i} t_{i}$.

(c) If $a, b \in R^{2}$, then $\lambda(a b)=(\lambda a) b=a(\lambda b)$.

Proof of Claim 2. (a) Suppose that $a=\sum_{i=1}^{n} x_{i} y_{i}=\sum_{i=1}^{n} x_{i}^{\prime} y_{i}^{\prime}$ and that $\lambda \bar{x}_{i}=\bar{z}_{i}$ and $\lambda \bar{x}_{i}^{\prime}=\bar{z}_{i}^{\prime}$ for each $i$. Then $\sum_{i=1}^{n} x_{i} y_{i}-\sum_{j=1}^{n} x_{j}^{\prime} y_{j}=0$; so by $\Phi_{4}$ we have $\sum_{i=1}^{n} z_{i} y_{i}-\sum_{j=1}^{n} z_{j}^{\prime} y_{j}=0$, and (a) follows.

(b) Suppose that $\bar{z}_{i}=\lambda \bar{x}_{i}$ for each $i$. Then by definition $\lambda a=\sum_{i=1}^{n} z_{i} y_{i}$; however, for each $i$ we also have $\bar{t}_{i}=\lambda\left(\bar{y}_{i}\right)$ and hence $z_{i} y_{i}=x_{i} t_{i}$ by $\Phi_{5}$. The result follows.

(c) Suppose that $a=\sum_{i=1}^{n} x_{i} y_{i}$ and that $\bar{z}_{i}=\lambda \bar{x}_{i}$ for each $i$. Then $a b=$ $\sum_{i=1}^{n} x_{i}\left(y_{i} b\right)$ and so by definition $\lambda a=\sum_{i=1}^{n} z_{i} y_{i}$ and $\lambda(a b)=\sum_{i=1}^{n} z_{i}\left(y_{i} b\right)$; hence $(\lambda a) b=\lambda(a b)$. The proof that $a(\lambda b)=\lambda(a b)$ is similar, but with (b) used instead of the definition of multiplication by $\lambda$.

It follows from Claim 2 that we may consider $R^{2}$ as a $\Lambda$-module; like the quotient $\bar{R}=R / A$ it is a finitely generated module by Zil'ber indecomposability. Hence both $R / A$ and $R^{2}$ are finite-dimensional spaces over $\mathfrak{K}$. Moreover $\left(A+R^{2}\right) / A$ is a $\Lambda$ submodule of $R / A$; let $W / A$ be a complementary $\mathfrak{K}$-subspace to $\left(A+R^{2}\right) / A$ in $R / A$. (It is in making this choice of $W$ that we lose definability, since we cannot assert that $\mathfrak{K}$ is definable in $\Lambda$.) Thus $W / A$ is finite-dimensional over $\mathfrak{K}$.

Now let $S$ be a complement to $R^{2} \cap A$ in $A$ and $T$ a complement to $A$ in $W$. Such complements exist because the subgroups are vector subspaces over $\mathbb{Q}$. Thus, using the canonical isomorphism between $\left(A+R^{2}\right) / R^{2}$ and $A /\left(A \cap R^{2}\right)$, we have $R=R^{2} \oplus S \oplus T$.

We can use the group isomorphism $T \cong W / A$ to make $T$ into a finite-dimensional vector space over $\mathfrak{K}$; so if $\lambda \in \mathfrak{K}$ and $x \in T$, then $\lambda x$ is defined by the two requirements that $A+\lambda x=\lambda(A+x)$ and $\lambda x \in T$.

By hypothesis, either $S$ is zero or $S$ has the same cardinality as $R / A$ and hence the same cardinality as $\mathfrak{K}$. In each case, we can make $S$ into a finite-dimensional vector space over $\mathfrak{K}$ in an arbitrary fashion.

The above definitions give $R$ the structure of a finite-dimensional vector space over $\mathfrak{K}$. We claim that $R$ is a $\mathfrak{K}$-algebra. To prove this, by distributivity it suffices to verify that $\lambda(x y)=(\lambda x) y=x(\lambda y)$ for all $x, y \in S \cup T \cup R^{2}$ and all $\lambda \in \mathfrak{K}$. If $x$ or 
$y$ is in $S$, this is clear since, $S$ being a subset of $A$, all products are 0 . If $x, y \in R^{2}$, the equalities follow from Claim 2 (c).

Next suppose that $x, y \in T$. From the definition of the $\Lambda$-module structure on $R^{2}$ we know that $\lambda(x y)=z y$ for any element $z \in R$ satisfying $A+z=\lambda(A+x)$; since $A+\lambda x=\lambda(A+x)$, we conclude that $\lambda(x y)=(\lambda x) y$. By Claim $2(\mathrm{~b}), \lambda(x y)$ is also equal to $x t$ for any $t$ such that $A+t=\lambda(A+y)$, and since $A+\lambda y=\lambda(A+y)$ we conclude that $\lambda(x y)=x(\lambda y)$.

By symmetry it will therefore suffice to prove that $\lambda(t s)=(\lambda t) s=t(\lambda s)$ for all $t \in T$ and all $s \in R^{2}$. The first equality follows directly from the above definition of the action of $\Lambda$ on $R^{2}$, and the second follows from Claim 2 (b).

\section{Proof of Theorem 1}

In order to apply Proposition 3.2, we need the following lemma.

Lemma 4.1. Let $G$ be a group viewed as an $\mathcal{L}$-structure where $\mathcal{L}$ is the language of groups, and let $H$ and $K$ be definable subgroups such that $K \leq H$. Assume that the coset spaces $G / H$ and $H / K$ are infinite. Then $G$ has an elementary extension $\tilde{G}$ such that $\tilde{G}, \tilde{G} / \tilde{H}$ and $\tilde{H} / \tilde{K}$ are of the same cardinality as $G$, where $\tilde{H}$ and $\tilde{K}$ are the subgroups defined by the formulas defining $H$ and $K$ in $G$.

Proof. This is an application of the compactness theorem. We start by adding constants to $\mathcal{L}$. More precisely, we define

$$
\mathcal{L}^{+}=\mathcal{L} \cup\left\{c_{i}, d_{i}: i<|G|\right\} \cup\left\{c_{g}: g \in G\right\} .
$$

Then we define

$$
\begin{aligned}
T^{+}= & \operatorname{Th}(G, g \in G) \cup\left\{c_{i} \in H: i<|G|\right\} \\
& \cup\left\{c_{i} c_{j}^{-1} \in K \text { if and only if } i=j\right\} \\
& \cup\left\{d_{i} d_{j}^{-1} \in H \text { if and only if } i=j\right\} .
\end{aligned}
$$

The theory $T^{+}$is finitely satisfiable in $G$ because $G / H$ and $H / K$ are infinite. Therefore by compactness, $T^{+}$has a model $\tilde{\mathcal{G}}^{+}$in which each of the cardinalities $\left|\tilde{\mathcal{G}}^{+} / \tilde{\mathcal{H}}^{+}\right|$and $\left|\tilde{\mathcal{H}}^{+} / \tilde{\mathcal{K}}^{+}\right|$is at least $|G|$. Since $\tilde{\mathcal{G}}^{+}$is an $\mathcal{L}^{+}$-structure and $\mathcal{L}^{+}$is of cardinality not higher than $|G|$, an application of the downward LöwenheimSkolem theorem implies that $T^{+}$has a model $\tilde{G}^{+}$of cardinality exactly $|G|$ with the corresponding subgroups $\tilde{H}^{+}$and $\tilde{K}^{+}$. The reduct $\tilde{G}$ of this model to $\mathcal{L}$ yields the desired structure.

Proof of Theorem 1. Let $G$ be a torsion-free nilpotent group of finite Morley rank. Suppose that $G$ has non-trivial definable normal subgroups $L_{1}, L_{2}$ with $L_{1} \cap L_{2}=1$. Then we write the trivial subgroup 1 as an irredundant intersection $K_{1} \cap \cdots \cap K_{m}$ of non-trivial definable normal subgroups with $m$ as large as possible; this can be done because $G$ has finite Morley rank, and in fact $m$ is at most the Morley rank of $G$. If we can prove that each $G / K_{i}$ can be embedded in $\mathrm{GL}_{n_{i}}\left(\mathfrak{K}_{i}\right)$ for some integer $n_{i}$ and field $\mathfrak{K}_{i}$ of characteristic 0 , then since $G$ can be embedded in $G / K_{1} \times \cdots \times G / K_{m}$, it follows that $G$ can be embedded in $\operatorname{GL}_{n}(\mathfrak{K})$, where $n=\sum_{i=1}^{m} n_{i}$ and $\mathfrak{K}$ is a field containing copies of all fields $\mathfrak{K}_{i}$. Since $G / K_{i}$ cannot have non-trivial definable normal subgroups with trivial intersection, it will therefore suffice to prove the result 
in the case where $G$ has a unique minimal definable normal subgroup. Moreover, since torsion-free abelian groups can be embedded in additive groups of fields, we can assume that $G$ is non-abelian. Finally, by Lemma 4.1 we can suppose in addition that $Z /\left(Z \cap G^{\prime}\right)$ has the same cardinality as $G / Z$, where $Z=Z(G)$ and $G^{\prime}$ is the derived group of $G$.

In this case it follows from Lemma 2.3 that the Lie algebra $L$ associated with $G$ in the Maltsev correspondence has a unique minimal definable ideal (necessarily contained in $\operatorname{Ann}(L))$ and has finite Morley rank; moreover $A /\left(A \cap L^{2}\right)$ has the same cardinality as $L / A$, where $A=\operatorname{Ann}(L)$. Therefore Proposition 3.2 is applicable, and we conclude that $L$ has the structure of a finite-dimensional algebra over a field $\mathfrak{K}$. By Lemma 2.3 and the remark following it, the group associated with $L$ by the Maltsev correspondence for Lie algebras over $\mathfrak{K}$ is (isomorphic to) $G$. Finally, $L$ can be regarded as a Lie algebra of triangular $n \times n$ matrices over $\mathfrak{K}$ for some $n$ by Ado's theorem ([1]; see also [8], pp. 202-203); then $G$ is isomorphic to the image of $L$ under the exponential map. Therefore $G$ can be embedded in $\mathrm{GL}_{n}(\mathfrak{K})$, and the result is proved.

\section{ACKNOWLEDGEMENTS}

Le premier auteur remercie Olivier Frécon pour des discussions éclairantes sur la question de linéarité. The first author would like to thank University College, Oxford, for providing ideal conditions during his stays at this institution.

The second author would like to thank the Forschungsinstitut für Mathematik at ETH, Zürich, where part of this work was carried out, for its hospitality and support.

\section{REFERENCES}

1. I. D. Ado. The representation of Lie algebras by matrices. Uspehi Mat. Nauk. (N.S.) 2 (1947), no. 6 (22), 159-173. (A.M.S Transl. No. 2 (1949)) MR.0027753 (10:350c)

2. A. Baudisch. A new uncountably categorical group. Trans. Amer. Math. Soc. 348 (1996), 3889-3940. MR1351488 (96m:03020)

3. A. V. Borovik and A. Nesin. Groups of Finite Morley Rank. Oxford University Press, 1994. MR.1321141 (96c:20004)

4. G. Cherlin and J. Reineke. Categoricity and stability of commutative rings. Ann. Math. Logic 10 (1976), 367-399. MR0480007 (58:208)

5. I. S. Cohen. On the structure and ideal theory of complete local rings. Trans. Amer. Math. Soc. 59 (1946), 54-106. MR0016094 (7:509h)

6. O. Frécon. Linearity of solvable groups of finite Morley rank. Preprint, 2007.

7. W. Hodges. Model Theory. Encyclopedia of Mathematics and its Applications 42. Cambridge University Press, 1993. MR.1221741 (94e:03002)

8. N. Jacobson. Lie Algebras. Dover Publications, 1962. MR0143793 (26:1345)

9. N. Jacobson. Basic Algebra II. W. H. Freeman and Company, 1989. MR.1009787(90m:00007)

10. A. Macintyre. On $\omega_{1}$-categorical theories of fields. Fund. Math. 71 (1971), 1-25. MR0290954 $(45: 48)$

11. A. Nesin. Non-associative rings of finite Morley rank. In The Model Theory of Groups, edited by A. Nesin and A. Pillay, Notre Dame Mathematical Lectures 11, Notre Dame Press, 1989, 117-137. MR.985343

12. I. Stewart. An algebraic treatment of Malcev's theorem concerning nilpotent Lie groups and their Lie algebras. Compositio Math. 22 (1970), 289-312. MR0288158(44:5356) 
13. R. B. Warfield Jr. Nilpotent Groups. Lecture Notes in Math. 513. Springer-Verlag, 1976. MR0409661 (53:13413)

14. B. I. Zil'ber. Rings with $\aleph_{1}$-categorical theories. Algebra and Logic 13 (1974), 95-104. (Translation from Algebra i Logika 13, No. 2 (1974), 168-187.) MR0366650 (51:2897)

Université de Lyon, Université Lyon 1, CNRS UMR 5208 Institut Camille Jordan, INSA de Lyon, F-69621, Ecole Centrale de Lyon, 43 Blvd du 11 novembre 1918, 69622 Villeurbanne cedex, France

E-mail address: altinel@math.univ-lyon1.fr

University College, Oxford OX1 4BH, United Kingdom

E-mail address: wilsonjs@maths.ox.ac.uk 